\title{
EXPLORATIONS FONCTIONNELLES
}

\section{Analyse des procédures du test de Hühner ou test post- coïtal tel qu'il est pratiqué en région Alsace}

\author{
A. Clatiert*, C. Cranz*,J-M. Gathrat**, D. Hachette ${ }^{\circ}$, D. Marx ${ }^{\circ}$, F. SchmitT“ \\ * CECOS Alsace, Laboratoire de Biologie de la Reproduction, Hôpitaux Universitaires de \\ Strasbourg, 67091 Strasbourg ; ** Laboratoire d'Analyses médicales du BOLLWERK, 4 \\ Avenue Salengro, 68100 Mulhouse ; ${ }^{\circ}$ Laboratoire de Biologie Médicale, 27 Rue du Général \\ de Gaulle 68240 Kaysersberg ; ${ }^{\circ}$ Laboratoire d'Analyses de Biologie Médicale KLUMP, 1 , \\ Rue Kuhn 67000 Strasbourg ; “ Laboratoire de Microbiologie de l'Hôpital E. Muller \\ (Moenschberg) 68100 Mulhouse.
}

Le test de Hühner est un test aisé, peu traumatisant, peu onéreux qui doit être réalisé en première intention dans tout bilan d'infertilité conjugale. Bien que son intérêt clinique et pronostic ait été très débattu du fait de nombreuses imprécisions dans sa réalisation et son interprétation, notre étude multicentrique montre qu'en fait il est bien standardisé. Cependant il apparaît une perte d'efficacité du fait d'une collaboration insuffisante entre prescripteur et biologiste.

Mots clés : Test de Hühner, test post coïtal, assurance de qualité.

Le test de Hühner ou test post coïtal consiste à étudier le mucus cervical en période ovulatoire après un rapport. C'est un test simple, non douloureux, peu onéreux qui doit être réalisé en première intention lors de l'exploration d'une infertilité conjugale. Le fait que l'intérêt clinique et pronostique de ce test ait été discuté par certains auteurs peut s'expliquer par des imprécisions trop fréquentes dans sa réalisation et son interprétation.

Il s'agit d'un test global qui permet d'apprécier l'étape initiale d'une succession d'évé- nements mettant en jeu les deux partenaires du couple et aboutissant à la fécondation. La qualité de ce test dépend effectivement de la sécrétion cervicale de la femme, de la qualité du sperme mais aussi de la qualité des rapports sexuels du couple.

S’il est positif, il est démontré que la sécrétion cervicale, la sécrétion oestrogenique, le sperme et le rapport sexuel sont dans les limites de la normale.

Par contre, s'il est négatif ou insuffisant, trois variables devront être considérées :

- la qualité de la glaire qui dépend elle même de la qualité intrinsèque des glandes endocervicales, de la qualité de la fonction ovarienne, de la date dans le cycle c'est à dire la plus ou moins grande proximité de l'ovulation à venir.

Sachant que la glaire n'est de qualité optimale que 48 heures environ avant l'ovulation [1], des résultats «faux négatifs « peuvent être enregistrés. Il apparaît là un paramètre important et parfois difficile à déterminer dans les cas de cycles irréguliers.

- la qualité du sperme que l'on ne connaît pas toujours au moment de l'examen 
- la technique coïtale: en effet, pour le couple, se voir imposer un rapport sexuel à un moment précis peut être mal vécu et le rapport sexuel devenir inefficace, ainsi les spermatozoïdes ne sont pas déposés au contact de la glaire d'où l'absence de spermatozoïdes dans celle-ci. Un interrogatoire permet dans certains cas, d'éviter une mauvaise interprétation.

Différents auteurs $[2,3]$ ont déjà publiés des recommandations pour la bonne pratique de ce test.

Localement nous avons réalisé ce travail pour connaître les pratiques des laboratoires d'analyses. Nous leur avons demandé de nous envoyer le double de tous les tests de Hühner réalisés en première intention. Ces laboratoires ont également accepté de répondre à un questionnaire et de faire des statistiques ponctuelles sur leur activité.

Nous tenons à remercier les laboratoires d'analyses médicales : LAM du Bollwerk (Mulhouse), LAM Brugiroux, Frage et Kientz (Haguenau), LAM du Diaconat (Mulhouse), LBM (Kaysersberg), LAM Klump (Strasbourg) et LAM des Vosges (Strasbourg) ainsi que le Laboratoire de Bactériologie (Hôpital du Moenschberg, Mulhouse) et le Laboratoire de Biologie de la Reproduction (HUS Strasbourg).

\section{METHODES}

Lors d'une réunion préparatoire, nous avons défini des critères communs en nous inspirant de ceux de l'OMS [4]. Pour le prélèvement, tous les laboratoires utilisent un Aspiglaire permettant de prélever la glaire de l'endocol vers l'exocol sans blesser la muqueuse endocervicale.

En ce qui concerne la glaire, les paramètres qualitatifs étudiés sont cotés de 0 à 3 selon la méthode du score d'Insler [5].

Nous avons également noté :

- la durée maximum et minimum des cycles,
- le jour du cycle du prélèvement,

- la durée de temps s'étant écoulé entre le prélèvement et le rapport sexuel (limite admise 3 à $12 \mathrm{~h}$ ),

Les paramètres étudiés sont les suivants :

- Les paramètres physiques de la glaire :

A. le volume de la glaire $(0,1(<0,25 \mathrm{ml}) 2$ $(0,25$ à $0,5 \mathrm{ml}) 3(>0,5 \mathrm{ml})$;

B. le $\mathrm{pH}$ évalué au papier $\mathrm{pH}(5,4-8,5)$;

C. La cristallisation caractérisée par l'aspect en feuille de fougère ( 0 absence, 1 ( 1 ramure), 2 (2 ramures), 3 (3 ramures);

D. La filance mesurée en $\mathrm{cm}: 0$ (absence), $1(<5 \mathrm{~cm}), 2(5$ à $8 \mathrm{~cm}), 3(>8 \mathrm{~cm})$.

- Le contenu cellulaire autre que les spermatozoïdes est noté :

E. Le nombre de cellules est évalué empiriquement de 0 à 3 . En période ovulatoire, il n'y a pas de cellules dans la glaire.

- La pénétration et la survie des spermatozoïdes est évaluée par :

F. La pénétration des spermatozoïdes (évaluée au grossissement $40 \mathrm{x}$ ), est évaluée par le nombre de spermatozoïdes observé par champ. (0,1 (1 - 5), 2 (5 - 15), 3 (> 15)) ;

G. La survie des spermatozoïdes étant le pourcentage de spermatozoïdes mobiles $(0,1(1-25 \%), 2(25-50 \%) 3(>50 \%)$.

\section{MATERIEL}

Nous avons pu étudier 115 dossiers dont 89 ont été retenus. Nous avons éliminé les tests faits chez des femmes aux cycles irréguliers ou supérieurs à 31 jours.

\section{RESULTATS}

\section{Analyse des résultats des tests :}

Sur 89 dossiers étudiés :

- Test bon : tous les paramètres supérieurs ou égaux à $2: 24$ soit $27 \%$ 
- Glaire dont tous les paramètres physiques $(\mathrm{A}, \mathrm{C}, \mathrm{D}, \mathrm{E})$ sont égaux ou supérieurs à 2 et $\mathrm{B}>7,4$ : Résultats permettant de qualifier la glaire de bonne 34 soit $38,2 \%$

- Glaire de bonne qualité avec une bonne survie $(F>2)$ mais pénétration des spermatozoïdes réduite $(\mathrm{G}<2) .10$ soit $11,3 \%$ de l'ensemble des test, soit $29,3 \%$ de l'ensemble des 34 glaires de bonne qualité.

- Glaires acides $(\mathrm{pH}<7$ avec les autres paramètres $(\mathrm{A}, \mathrm{Cr} \mathrm{D}, \mathrm{E})$ égaux ou supérieurs à 220 soit $22,5 \%$ du total dans 17 cas il y a absence de survie des spermatozoïdes dans 3 cas la survie est très réduite.

\section{Analyse de la pratique du test}

La mesure du $\mathrm{pH}$ endocervical est homogène d'un laboratoire à l'autre ; par contre, le $\mathrm{pH}$ exocervical est très variable. Cette variabilité est liée au fait que certains laboratoires procèdent à une toilette vaginale avant de prélever alors que d'autres ne le font pas.

- Dans 50\% des cas, les laboratoires ne sont pas en possession des conclusions du spermogramme, ce qui peut rendre l'interprétation du résultat difficile.

- Jour du prélèvement.

Il est variable d'un laboratoire à l'autre. Les valeurs extrêmes des moyennes par laboratoire sont 13,06 et 14,13 .

\section{- Suivi du test}

Trois laboratoires ont pu étudier leur dossier et suivre l'évolution des examens demandés par la suite, les résultats sont identiques d'un laboratoire à l'autre :

- dans 14\% des cas, le test est suivi par un test de contrôle,

- dans $1,5 \%$ des cas, par un second contrôle.

\section{DISCUSSION}

Dans $27 \%$ des cas,le test de Hühner permet, dans les conditions que nous avions fixées, de conclure que le sperme, la glaire et le rapport sont efficaces. Dans ces conditions les causes de stérilité sont autres et une nouvelle orientation de l'exploration peut être donnée.

Dans $11,3 \%$ des cas, la glaire est de bonne qualité, avec une bonne survie mais avec une pénétration réduite. Cette situation s'explique uniquement par une oligospermie importante et nécessite une exploration andrologique.

Dans notre population, le facteur andrologique intervient pour environ $30 \%$ si l'on rapporte ces 10 cas aux 34 cas de la glaire de bonne qualité fonctionnelle.

Dans les cas où les paramètres physico-chimiques sont bons sauf le $\mathrm{pH}$ qui est inférieur à 7 , la survie des spermatozoïdes est toujours réduite ou nulle. Ces résultats sont cohérents car on sait que le spermatozoïde ne résiste pas à une telle acidité du milieu. L'acidité de la glaire peut s'expliquer soit par la multiplication des lactobacilles secondaire à un déséquilibre œestroprogestatif, soit par une infection.

Ce chiffre montre combien les facteur cervicaux vaginaux sont importants dans l'hypofertilité et la stérilité féminine.

En pratique, l'analyse des résultats quantitatifs montre une grande homogénéité que ce soit entre les laboratoires ou au sein de chaque laboratoire. Ainsi cet examen peut être facilement normalisé et les résultats peuvent atteindre rapidement une cohérence suffisante pour réaliser des études épidémiologiques .

Quelques précautions particulières, notamment la réalisation d'une toilette vaginale, réalisée à l'aide d'une compresse sèche, permettra aisément d'harmoniser les résultats. 
Cependant des résultats de cette étude, il apparaît que le moment de la réalisation du test qui est en général fixé par le médecin prescripteur, pose un problème qu'il convient de résoudre. L'existence de faux négatifs est liée à la réalisation trop tardive du test. En effet on sait que la sécrétion maximum de la glaire a lieu pendant un délai de 48 à 72 heures précédant l'ovulation, et c'est à ce moment que le test doit être réalisé ce qui n'est pas la pratique courante puisque la moyenne se situe autour de 14 jours pour des cycles inférieurs ou égaux à 30 jours. Depuis la FIV qui se réalise au moment de l'ovulation les gynécologues prescripteurs ont pris l'habitude de se repérer préférentiellement à ce moment. Adresser le couple plus tôt dans le cycle et autoriser le biologiste à refaire l'examen s'il estime être trop loin de l'ovulation permettrait aux gynécologues d'être plus efficaces dans l'exploration, en évitant d'être obligé de réaliser des contrôles lors des cycles suivants.

En outre l'interprétation du test de Hühner s'il n'est pas normal est difficile en l'absence de renseignements concernant la qualité du sperme. Il serait souhaitable de proposer au couple de réaliser un spermogramme dès le début de l'exploration.

Par ailleurs nous avons été frappé par un fait: $62 \%$ des test de Hühner initiaux sont de mauvaise qualité, or seuls $14 \%$ des tests pratiqués sont à nouveau contrôlés lors d'un cycle suivant. Ceci peut correspondre au fait qu'un autre facteur d'hypofertilité a été diagnostiqué, en général une dysovulation, et que de ce fait le facteur cervical soit relégué au second plan. Un traitement inducteur de l'ovulation est alors instauré, normalisant l'ovulation, mais sans vérification des effets de la thérapeutique sur la qualité de la glaire. Cette pratique peut être néfaste pour l'efficacité des traitements car des facteurs cervicaux et / ou vaginaux peuvent persister malgré la normalisation de l'ovulation.

\section{CONCLUSION}

Le test de Huhner ou test post coïtal est bien normalisé quant à son analyse biologique mais il existe une perte d'efficacité par une collaboration insuffisante entre prescripteur et biologiste

\section{REFERENCES}

1. .Schwartz, D, Mayaux M.-J., Heuche V., Czyglik F. ET DAVID G. : Importance of insemination timing and frequency in AID. in: Human artificial insemination and semen preservation David G et Ed. Plenum Press. 1980 : 333-340.

2. Belaisch J. : Le test post coïtal chez la femme in: Période péri ovulatoire, Salat-Baroux et Thibault Ed. Masson. 1984 : 85-91.

3. LaVAUd M.C. : Le test post coïtal. Andrologie. 1994, $4,346-352$.

4. WHO : Laboratory normal for the examination of the human semen and sperm mucus interaction. Ed. CambridgeUniversityPress. 1992

5. Insler V., Melmed H., Eichenhrenner L., SerR D.M., LUNNENFELD B. : The cervical score. A simple semi quantitative method fbr monitoring of the menstrual cycle. Inter. Journal of Gynecol. and Obst. 1972 : 10: 223-28.

\section{ABSTRACT}

\section{A. Clavert, C. Cranz et al.}

The Huhner test is an easy, unpainful, unexpensive test which must be done first at the time of unfertility exploration.

His clinical and prognosticated interest is much debated because many imprecisions in its realisation and interpretation occur. Our multicentric study proves that this test is quite standardized in his realisation but a loss of its efficacity appears by the fact of a inadequate collaboration between attending physicians and biologists.

Key Words : Post coïtal test. 